\title{
L-band Q-switched fiber laser with gallium/thulium-doped silica fiber saturable absorber
}

\begin{abstract}
An L-band Q-switched fiber laser was demonstrated using gallium/thulium-doped silica fiber saturable absorber. At $10 \mathrm{~cm}$ in length, the saturable absorber generated Q-switched fiber laser at a pump power threshold of $39.6 \mathrm{~mW}$ and a central wavelength of $1601.93 \mathrm{~nm}$. Beyond 53.3 $\mathrm{mW}$, a spectrum with a central wavelength at $1602.00 \mathrm{~nm}$ was generated. On the other hand, the pulse repetition rate was obtained from 3.44 to $7.47 \mathrm{kHz}$ whereas the pulse width reduces from 100.2 to $58.6 \mu$ s for pump power ranges from 39.6 to $53.3 \mathrm{~mW}$. Within this range, the pulse energy is attained between 0.2600 and $0.2843 \mu \mathrm{J}$, at a laser power slope efficiency of $6.94 \%$. The constantly operated Q-switched fiber laser over $50 \mathrm{~min}$ observation time at 53.3 $\mathrm{mW}$ pump power ensures the feasibility of this pulse laser source as a practical device.
\end{abstract}

Keyword: Q-switching; Fiber laser; Gallium; Thulium-doped fiber; Saturable absorber 